\title{
Observer-based controllers with data dropout rate adaptation
}

\author{
Daniel Dolz, Ignacio Peñarrocha*, Roberto Sanchis \\ Departament d'Enginyeria de Sistemes Industrials i Disseny, Universitat Jaume I, 12071 Castelló, Spain.
}

\begin{abstract}
SUMMARY
In this work, we address the observer-based control problem for networked control systems with an unknown time-varying packet arrival rate (PAR) and under RMS-norm bounded disturbances. We assume packetized transmissions of both measurement and control input through a communication network with successful delivery acknowledgement. Using the measurement reception state and the control transmission acknowledgement, we derive a filter to estimate the PAR. We consider that the PAR changes sporadically from a constant value to another one, i.e., it has two different behaviours: transient and steady state. While the observer only updates the state estimation using the current received measurements, the controller computes the control action employing the current state estimation and the previous applied control input. We propose to schedule both the observer and controller with rational functions of the PAR estimation. We show that the separation principle applies and then, seeking higher performance accuracy, we develop an optimization $H_{\infty}$ observer and controller design procedure that considers the two possible behaviours of the PAR. This optimization procedure attempts to maximize the estimation and control performances for each of the possible constant values of the PAR while offering robustness against PAR estimation errors and variations of the PAR. Exploiting sum-of-squares (SOS) decomposition techniques, the design procedure involves an optimization problem over polynomials. A numerical example illustrates the effectiveness of the proposed approach. Copyright (c) 2015 John Wiley \& Sons, Ltd.
\end{abstract}

Received ...

KEY WORDS: Networked control systems, observer-based control, time-varying data rate, gainscheduling, sum-of-squares.

\section{INTRODUCTION}

Networked control systems (NCSs) are characterized by the exchange of control and feedback information among the elements of the control architecture through a communication network. The use of a network increases flexibility and ease of manoeuvre at the cost of offering an unreliable communication link subject to, e.g., dropouts and time delays [1]. Thus, when controlling over a network one must guarantee in addition, robustness against these network-induced problems.

One common way to model the stochastic behaviour of the data loss process is by a finite Markov chain [2]. The transition probabilities have been widely considered to be known a priori. However, in real networked applications this is an unusual situation since it can be difficult to get the exact value. As a result of this, much attention has been focused recently on dealing with uncertain transition probabilities when designing networked controllers on a robust control framework [3-5]. Among these works, one might give special attention to [3] where the authors unified the three different types of considered uncertainties in the probabilities: partly unknown, bounded and polytopic. Nevertheless, they all assumed time-invariant probabilities, and this limits the application scope since the packet arrival rate (PAR) can also change due to many uncontrollable and unforeseen

${ }^{*}$ Correspondence to: Ignacio Peñarrocha, Departament d'Enginyeria de Sistemes Industrials i Disseny, Universitat Jaume

I, 12071 Castelló, Spain. E-mail: ipenarro@uji.es 
reasons. For instance, the connection of new elements to the network may congestion it, decreasing the PAR. Also in wireless networks, the PAR is directly affected by the channel fading gains, which are stochastic phenomena [6]. Therefore, it seems to be a gap between the previous works that only address probability uncertainty issues and those which only focus on its time-varying nature $[7,8]$. In this work, we aim to tackle both problems at the same time by considering, as a first approach, that packetized transmissions are subject to dropouts following a Bernoulli distribution (this model has often been employed, see $[9,10])$ with successful delivery acknowledgement.

Works such as $[2,11]$ proposed the use of jump strategies for the networked state estimation and control problem whose modes are related to the transmission outcome history in order to imitate the behaviour of optimal on-line solutions [12], which in general are not suitable under RMS-norm bounded disturbances. In this aim, one might think of deriving a PAR estimator and scheduling the observer and controller gains with an inexact parameter, the estimated PAR. Scheduling procedures with inexact parameters are an increasingly appealing solution to control linear parameter varying (LPV) systems that improve performance [13-16]. LPV systems have been used to model NCS, but while the previous works scheduled the controller architecture with an uncertain measurement of the governing parameters, here we seek instead to tune the control strategy with the stochastic behaviour of a network parameter, the PAR. Probability-dependent approaches were already analysed in [17-19] but assuming that the probability was perfectly known. Therefore, designing observer-based controllers scheduled with an inexact PAR is still a challenging problem.

In the current paper, using the measurement and control input transmission outcomes at each time instant, we derive a first order filter to estimate in real time the uncertain time-varying PAR, which can vary arbitrarily fast (different from [20], where, in addition, only the state estimation problem was considered). Also, we characterize the PAR estimation error by obtaining the maximum and minimum values during any kind of network behaviour (transient or steady state), and by using a probabilistic domain when the PAR is on steady state. Then, we propose the use of a Luenbergertype state observer and a controller that uses both the current state estimation and previous applied control input (c.f. [15]) whose gains are scheduled with the real time PAR estimation. Under this observer-based control approach, the separation principle holds. Assuming an arbitrary rate of change of the PAR leads to a highly robust solution with nearly PAR independent gains, see [18-20]. To reduce conservativeness, we introduce a novel $H_{\infty}$ design methodology based on parameter dependent matrix inequalities (PDMIs) that focuses on performance when the PAR is constant while guarantees robustness against PAR variations and PAR estimation errors. We propose the use of SOS decomposition techniques [21] to address the parametrized optimization design problem.

Notation : Let $\mathbb{R}$ and $\mathbb{N}$ denote the real and natural numbers set. Let $A$ and $B$ be some matrices. $A \preceq 0$ means that matrix A is negative semidefinite. Similar applies to $\prec, \succ$ and $\succeq$. The direct sum is represented as $\bigoplus$, where $A \bigoplus B$ is a block diagonal matrix with $A$ and $B$ on its diagonal. $\mathbf{E}\{\cdot\}$ and $\operatorname{Pr}\{\cdot\}$ denotes expectation and probability. We denote the RMS norm of process $x_{k} \in \mathbb{R}^{n}$ as $\|x\|_{\mathrm{RMS}}=\lim _{K \rightarrow \infty} \sqrt{\frac{1}{K} \sum_{k=0}^{K-1} x_{k}^{T} x_{k}}$.

\section{PROBLEM SETUP}

We consider the remote control problem of linear time-invariant discrete-time system over networks with packet successful delivery acknowledgement subject to dropouts, see Fig. 1. We describe the system in its state-space form as:

$$
\begin{aligned}
& x_{k}=A x_{k-1}+B_{u} u_{k-1}+B_{w} w_{k-1}, \\
& y_{k}=C x_{k}+v_{k},
\end{aligned}
$$

where $x_{k} \in \mathbb{R}^{n}$ is the state at instant $k \in \mathbb{N}, u_{k} \in \mathbb{R}^{n_{u}}$ is the control input, $w_{k} \in \mathbb{R}^{n_{w}}$ is the unmeasurable state disturbance, $y_{k} \in \mathbb{R}^{n_{y}}$ is the measured output and $v_{k} \in \mathbb{R}^{n_{y}}$ is the measurement noise. We also consider that both state disturbances and measurement noises are bounded such as $\|w\|_{\mathrm{RMS}} \leq \bar{w}_{\mathrm{rms}}$ and $\|v\|_{\mathrm{RMS}} \leq \bar{v}_{\text {rms }}$ (not necessarily Gaussian). 


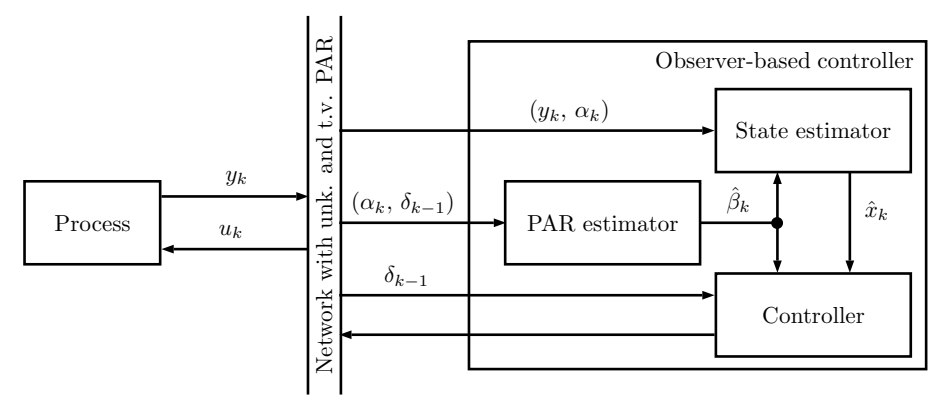

Figure 1. Networked control architecture under an unknown and time-varying PAR.

\subsection{Networked transmission description}

At each instant $k$, each sensor samples its associated output. Then, the measurements are aggregated in a single time-stamped packet $y_{k}$ that is sent to the observer-based controller through a communication network subject to packet dropouts. We model the packetized measurement reception at instant $k$ with the binary variable

$$
\alpha_{k}= \begin{cases}1 & \text { if } y_{k} \text { is received at instant } k \\ 0 & \text { if } y_{k} \text { is lost. }\end{cases}
$$

With the received information, the observer-based controller computes at instant $k$ the control commands and transmits them within a single packet $u_{k}$ to the actuators, which implement a generalized hold-input strategy (see [22]). We describe the control input reception at instant $k$ with

$$
\delta_{k}= \begin{cases}1 & \text { if } u_{k} \text { is received at instant } k \\ 0 & \text { if } u_{k} \text { is lost. }\end{cases}
$$

We assume that $\left\{\alpha_{k}\right\}$ and $\left\{\delta_{k}\right\}$ are independent non-stationary stochastic processes with the same distribution, i.e.,

$$
\beta_{k}=\operatorname{Pr}\left\{\alpha_{k}=1\right\}=\operatorname{Pr}\left\{\delta_{k}=1\right\}, \quad \beta_{\min } \leq \beta_{k} \leq 1 .
$$

We consider that the behaviour of the packet arrival rate (PAR) $\beta_{k}$ is described by two possible modes: a transient mode where the PAR is time variant, i.e., $\beta_{k} \neq \beta_{k-1}$, and a steady state mode where the PAR is constant, i.e., $\beta_{k}=\beta_{k-1}$. In contrast with most of the existing works in the networked control system literature, we consider that $\beta_{k}$ is not directly accessible. While the real PAR is unknown, at time $k$ the observer-based controller has access to the outcomes of the measurement transmissions at the current instant $\alpha_{k}$, simply by checking the acquisition of new measurement packets, as well as to the status of successfully delivered control inputs at the previous instant $\delta_{k-1}$, which is provided by an acknowledgement protocol implemented by the network. Making use of this information, we propose the use of a first order filter (exponential smoothing technique), which is simple to implement, to estimate the PAR (focusing on the network steady state behaviour) such as

$$
\hat{\beta}_{k}=a \hat{\beta}_{k-1}+(1-a)\left(\alpha_{k}+\delta_{k-1}\right) / 2
$$

where $0<a \lesssim 1^{\dagger}$ is a tuning parameter representing a trade-off between a fast adaptation to probability changes and a low error variance estimation under constant probabilities.

\footnotetext{
$\overline{{ }^{\dagger} \text { The expression } 0}<a \lesssim 1$ means that $a$ is between 0 and 1 but closer to 1 .
} 
Let us define the PAR estimation error by $\tilde{\beta}_{k}=\beta_{k}-\hat{\beta}_{k}$. In the general case (time-varying or constant PAR), the estimation algorithm could produce a maximum instantaneous error bounded by

$$
\tilde{\beta}_{k} \in\left[\beta_{k}-1, \beta_{k}\right] \text {. }
$$

Assuming a steady state behaviour of the network with a time-invariant PAR, i.e., $\beta_{k}=\beta_{k-1}=\bar{\beta}$ for all $k$, the PAR estimation error has the properties indicated in the following lemma.

Lemma 2.1

If $\beta_{k}=\beta_{k-1}=\bar{\beta}$, the estimation error $\tilde{\beta}_{k}$ has the following properties in steady state

$$
\mathbf{E}\left\{\tilde{\beta}_{k}\right\}=0, \quad \mathbf{E}\left\{\tilde{\beta}_{k}^{2}\right\}=\frac{1-a}{2(1+a)} \bar{\beta}(1-\bar{\beta}) .
$$

\section{Proof}

If the PAR is constant, we have $\beta_{k-1}=\bar{\beta}$ and thus, $\left\{\alpha_{k}\right\} \in\{0,1\}$ and $\left\{\delta_{k}\right\} \in\{0,1\}$ follow a Bernoulli distribution where $\mathbf{E}\left\{\alpha_{k}\right\}=\mathbf{E}\left\{\delta_{k}\right\}=\mathbf{E}\left\{\alpha_{k}^{2}\right\}=\mathbf{E}\left\{\delta_{k}^{2}\right\}=\bar{\beta}, \quad \mathbf{E}\left\{\alpha_{k} \delta_{k}\right\}=\bar{\beta}^{2}$. Let us define the random variable $\eta_{k}=\bar{\beta}-\alpha_{k} / 2-\delta_{k-1} / 2$. Then $\left\{\eta_{k}\right\} \in[\bar{\beta}-1, \bar{\beta}]$ is a bounded sequence of random variables such as

$$
\mathbf{E}\left\{\eta_{k}\right\}=0, \quad \mathbf{E}\left\{\eta_{k}^{2}\right\}=\mathbf{E}\left\{\left(\bar{\beta}-\alpha_{k} / 2-\delta_{k-1} / 2\right)^{2}\right\}=\frac{1}{2} \bar{\beta}(1-\bar{\beta}) .
$$

From (5), the dynamics of the estimation error with constant PAR reduces to

$$
\tilde{\beta}_{k}=a \tilde{\beta}_{k-1}+(1-a) \eta_{k} .
$$

Since $a \in(0,1)$, the previous recursion converges to a stationary stochastic distribution. Under the steady state, taking expectations in both sides of recursion (9) we obtain that $\mathbf{E}\left\{\tilde{\beta}_{k}\right\}$ is zero, where we have considered (8) and that $\mathbf{E}\left\{\tilde{\beta}_{k}\right\}=\mathbf{E}\left\{\tilde{\beta}_{k-1}\right\}$. Now, performing the expectation of the square of both sides of recursion (9), and taking into account that $\mathbf{E}\left\{\tilde{\beta}_{k}^{2}\right\}=\mathbf{E}\left\{\tilde{\beta}_{k-1}^{2}\right\}, \mathbf{E}\left\{\tilde{\beta}_{k-1} \eta_{k}\right\}=0$ and (8), we obtain the $\mathbf{E}\left\{\tilde{\beta}_{k}^{2}\right\}$ as in (7).

\section{Remark 2.1}

While in [20] the PAR was supposed to change slowly and to be estimated with a low error, in this work we do not make any assumption neither on the PAR rate of change nor on the estimation error. The idea here is to make the procedure more general and to obtain a less conservative result during the intervals of time when the PAR remains constant. If the PAR has step-like changes due to events such as the connection of new devices, the transient number of samples needed to reach an average estimation of the PAR with an error less than $2 \%$ will be in the order of $\frac{\log (0.02)}{\log (a)}$. Therefore, $a$ represents the trade-off between PAR estimation accuracy during steady state and transient adaptation, i.e., higher values (near 1) are suitable for sporadic PAR changes, while lower values allow to follow faster frequent PAR variations.

\section{OBSERVER-BASED CONTROL STRATEGY}

In this work we focus on observer-based controllers where both observer and controller are collocated. Considering the given measurement transmission description in Section 2.1, the state estimation algorithm is

$$
\begin{aligned}
\hat{x}_{k^{-}} & =A \hat{x}_{k-1}+B_{u} u_{k-1}, \\
\hat{x}_{k} & =\hat{x}_{k^{-}}+L_{k} \alpha_{k}\left(y_{k}-C \hat{x}_{k^{-}}\right) .
\end{aligned}
$$

At instant $k$, we compute an open loop state estimation $\hat{x}_{k^{-}}$using (10a). Then, when possible, we correct the estimation with the received measurements (when $\alpha_{k}$ is 1 ) through the gain matrix $L_{k}$, 
see (10b). Defining the state estimation error as $\tilde{x}_{k}=x_{k}-\hat{x}_{k}$, its dynamic is given by

$$
\tilde{x}_{k}=\left(I-\alpha_{k} L_{k} C\right)\left(A \tilde{x}_{k-1}+B_{w} w_{k-1}\right)-\alpha_{k} L_{k} v_{k} .
$$

Within the presented control input transmission framework, at instant $k$, the controller computes and sends a new control input by means of the current state estimation and the previous used control command, which are compensated through gains $K_{k}$ and $F_{k}$, respectively. If the transmitted control input is lost (i.e., $\delta_{k}=0$ ), then a weighted previous value is applied to the process (generalized hold-input strategy [22]). Thus, the control action being applied at the system is

$$
u_{k}= \begin{cases}K_{k} \hat{x}_{k}+F_{k} u_{k-1} & \text { if } \delta_{k}=1, \\ \mu u_{k-1} & \text { if } \delta_{k}=0 .\end{cases}
$$

where $\mu \in[0,1]$.

\subsection{Closed loop analysis}

Considering the system defined by (1), the estimation algorithm given by (10), the resulting state estimation error in (11) and the applied control command in (12), the closed loop dynamic equations are

$$
\begin{aligned}
{\left[\begin{array}{c}
x_{k+1} \\
u_{k} \\
\tilde{x}_{k+1}
\end{array}\right] } & =A_{k+1}^{C L}\left[\begin{array}{c}
x_{k} \\
u_{k-1} \\
\tilde{x}_{k}
\end{array}\right]+B_{k+1}^{C L}\left[\begin{array}{c}
w_{k} \\
v_{k+1}
\end{array}\right], \\
y_{k} & =\left[\begin{array}{lll}
C & 0 & 0
\end{array}\right]\left[\begin{array}{c}
x_{k} \\
u_{k-1} \\
\tilde{x}_{k}
\end{array}\right]+v_{k},
\end{aligned}
$$

where

$$
\begin{aligned}
A_{k+1}^{C L} & =\left[\begin{array}{ccc}
A+\delta_{k} B_{u} K_{k} & \delta_{k} B_{u} F_{k}+\left(1-\delta_{k}\right) \mu B_{u} & -\delta_{k} B_{u} K_{k} \\
\delta_{k} K_{k} & \delta_{k} F_{k}+\left(1-\delta_{k}\right) \mu & -\delta_{k} K_{k} \\
0 & 0 & \left(I-\alpha_{k+1} L_{k+1} C\right) A
\end{array}\right], \\
B_{k+1}^{C L} & =\left[\begin{array}{cc}
B_{w} & 0 \\
0 & 0 \\
\left(I-\alpha_{k+1} L_{k+1} C\right) B_{w} & -\alpha_{k+1} L_{k+1}
\end{array}\right] .
\end{aligned}
$$

The authors of $[23,24]$ proved that the separation principle holds for networked control systems with a triangular structure such as the one that has the dynamic matrix $A_{k+1}^{C L}$. This implies that the observer and controller can be designed independently to achieve a mean square stable behaviour, and thus guaranteeing the mean square stability of the closed loop system. Note that, the estimation error can be considered as a new bounded disturbance affecting the controller, whose upper bound comes from the observer design.

\subsection{Gain-scheduling approach}

In this work we aim to obtain state observer gain matrix $L_{k}$, controller gain matrices $K_{k}$ and $F_{k}$, and PAR estimator tuning parameter $a$ such that we guarantee mean square stability and some attenuation levels from state disturbance and noise measurement for any possible values of $\beta_{k}$.

When dealing with the state estimation problem, jump linear estimators that relate their modes to the measurement reception outcome history improve estimation performances with respect to employing invariant gain estimators [2,25]. Instead of focusing on the reception outcomes, some works as $[19,20]$ proposed probability-dependent gain scheduled observers to enhance estimation performances. Recently, this approach was extended in [18] to the state feedback control problem. In this aim, we propose a gain-scheduled observer-based control approach depending on the estimation 
of the PAR, i.e.,

$$
L_{k}=L\left(\hat{\beta}_{k}\right), \quad K_{k}=K\left(\hat{\beta}_{k}\right), \quad F_{k}=F\left(\hat{\beta}_{k}\right) .
$$

Considering a PAR that varies arbitrarily fast over time, i.e. without any bound in the rate of change, would lead to conservative observers [19,20] or controllers [18] with gains that are almost independent of the PAR (i.e., robust to the variation of the PAR). In the aim of reducing conservativeness, we propose an alternative $H_{\infty}$ design method that takes into account the two different behaviours of the PAR: transient $\left(\beta_{k} \neq \beta_{k-1}\right)$ and steady state $\left(\beta_{k}=\beta_{k-1}=\bar{\beta}\right)$. Then, we will design both observer and controller in such a way that: i) for a general PAR scenario (including transient and steady state), we guarantee mean square stability for all the possible values of $\left\{\beta_{k}, \tilde{\beta}_{k}\right\}$ and disturbances, ii) when the PAR is constant, for each $\bar{\beta}$ we minimize the impact of the disturbances on the system performance for all possible values of $\tilde{\beta}_{k}$.

\section{Remark 3.1}

A different observer-based control approach under data dropout was considered in $[22,26]$ where the authors proposed a modified version of a Kalman filter plus a LQG controller. Although that controller might work under the studied networked scenario, it is limited to Gaussian noises. Here we consider a wider class of disturbances (bounded in RMS norm and non necessarily independent of the state of the system) which could also represent model uncertainties. Moreover, using predefined gains alleviates the implementation computational burden [25].

\section{STATE ESTIMATOR DESIGN}

In this section we study the design of the gain-scheduled observer with law $L\left(\hat{\beta}_{k}\right)$ by means of sufficient conditions that guarantee the design goals stated in Section 3.2. We shall first analyse the case when the PAR might arbitrarily vary on its domain, then the case when the PAR is constant and finally we will propose and optimization-based design procedure for any occurrence of the PAR. All the conditions are given by means of PDMIs.

The next theorem states under which conditions the state estimation error is bounded subject to a general PAR scenario (including the steady state case) with any possible PAR estimation error.

Theorem 4.1

Consider the state estimation algorithm (10) applied to system (1) and the PAR estimation algorithm (5). If there exist positive definite matrices $P^{o}\left(\beta_{k}\right) \in \mathbb{R}^{n \times n}$, full matrices $G^{o}\left(\hat{\beta}_{k}\right) \in \mathbb{R}^{n \times n}$ and $X^{o}\left(\hat{\beta}_{k}\right) \in \mathbb{R}^{n \times n_{y}}$, and positive scalars $\gamma_{w}^{o} \in \mathbb{R}$ and $\gamma_{v}^{o} \in \mathbb{R}$, fulfilling ${ }^{\ddagger}$

$$
M^{o}=\left[\begin{array}{ccc}
M_{11}^{o} & M_{12}^{o} & M_{13}^{o} \\
\star & M_{22}^{o} & M_{23}^{o} \\
\star & \star & M_{33}^{o}
\end{array}\right] \succ 0, \quad \forall\left\{\beta_{k}, \beta_{k-1}, \hat{\beta}_{k}\right\} \in \mathcal{S}_{1} \times \mathcal{S}_{1} \times \mathcal{S}_{2}
$$

with

$$
\begin{aligned}
& M_{11}^{o}=\beta_{k}\left(G^{o}\left(\hat{\beta}_{k}\right)+G^{o}\left(\hat{\beta}_{k}\right)^{T}-P^{o}\left(\beta_{k}\right)\right), \quad M_{12}^{o}=\beta_{k}\left(G^{o}\left(\hat{\beta}_{k}\right)-X^{o}\left(\hat{\beta}_{k}\right) C\right) A, \\
& M_{13}^{o}=\left[\beta_{k}\left(G^{o}\left(\hat{\beta}_{k}\right)-X^{o}\left(\hat{\beta}_{k}\right) C\right) B_{w}-\beta_{k} X^{o}\left(\hat{\beta}_{k}\right)\right] \\
& M_{22}^{o}=P^{o}\left(\beta_{k-1}\right)-\left(1-\beta_{k}\right) A^{T} P^{o}\left(\beta_{k}\right) A-I, \\
& M_{23}^{o}=\left[\begin{array}{ll}
-\left(1-\beta_{k}\right) A^{T} P^{o}\left(\beta_{k}\right) B_{w} & 0
\end{array}\right], \quad M_{33}^{o}=\left(\gamma_{w}^{o} I-\left(1-\beta_{k}\right) B_{w}^{T} P^{o}\left(\beta_{k}\right) B_{w}\right) \oplus \gamma_{v}^{o} I,
\end{aligned}
$$

and

$$
\mathcal{S}_{1}=\left\{\beta_{k}: \beta_{\min } \leq \beta_{k} \leq 1, \forall k\right\}, \quad \mathcal{S}_{2}=\left\{\hat{\beta}_{k}: 0 \leq \hat{\beta}_{k} \leq 1, \forall k\right\}
$$

$\ddagger_{\star}$ stands for the corresponding block matrix assuring symmetry. 
then, defining the observer gain matrices as $L\left(\hat{\beta}_{k}\right)=G^{o}\left(\hat{\beta}_{k}\right)^{-1} X^{o}\left(\hat{\beta}_{k}\right)$ : i) in the absence of disturbances and measurement noises the average state estimation error (11) converges to zero, ii) under zero initial conditions, the state estimation error is bounded by

$$
\mathbf{E}\left\{\|\tilde{x}\|_{\mathrm{RMS}}^{2}\right\}<\gamma_{w}^{o} w_{\mathrm{rms}}^{2}+\gamma_{v}^{o} v_{\mathrm{rms}}^{2} .
$$

Proof

Sets $\mathcal{S}_{1}$ (domain of $\beta_{k}$ and $\beta_{k-1}$ ) is derived from the definition in (4), while $\mathcal{S}_{2}$ (domain of $\hat{\beta}_{k}$ ) is obtained considering (5). If (16) holds, the PDMI resulting after replacing $M_{11}^{0}=$ $\beta_{k} G^{o}\left(\hat{\beta}_{k}\right) P^{o}\left(\beta_{k}\right)^{-1} G^{o}\left(\hat{\beta}_{k}\right)$ is also positive definite since $\left(P^{o}\left(\beta_{k}\right)-G^{o}\left(\hat{\beta}_{k}\right)\right) P^{o}\left(\beta_{k}\right)^{-1}\left(P^{o}\left(\beta_{k}\right)-\right.$ $\left.G^{o}\left(\hat{\beta}_{k}\right)\right)^{T} \succeq 0$, where $P^{o}\left(\beta_{k}\right) \succ 0$ by definition. With this in mind, if we define the Lyapunov function as $V_{k}=V\left(\tilde{x}_{k}, \beta_{k}\right)=\tilde{x}_{k}^{T} P^{o}\left(\beta_{k}\right) \tilde{x}_{k}$, applying a congruence transformation on (16) by matrix $G^{o}\left(\hat{\beta}_{k}\right)^{-1} \oplus I \oplus I$, taking Schur's complements and premultiplying the result by $\left[\begin{array}{lll}\tilde{x}_{k-1}^{T} & w_{k-1}^{T} & v_{k}^{T}\end{array}\right]$ and postmultiplying by its transpose leads to

$$
\mathbf{E}\left\{V_{k}\right\}-V_{k-1}+\tilde{x}_{k}^{T} \tilde{x}_{k}-\gamma_{w}^{o} w_{k-1}^{T} w_{k-1}-\gamma_{v}^{o} v_{k}^{T} v_{k}<0 .
$$

for all $\left\{\beta_{k}, \beta_{k-1}, \hat{\beta}_{k}\right\} \in \mathcal{S}_{1} \times \mathcal{S}_{1} \times \mathcal{S}_{2}$.

i) In the absence of disturbances $\left(w_{k-1}=0\right)$ and measurement noises $\left(v_{k}=0\right)$, (20) leads to $\mathbf{E}\left\{V_{k+1}\right\}-V_{k}<0$ which guarantees that the average state estimation error converges to zero for all $\left\{\beta_{k}, \beta_{k-1}, \hat{\beta}_{k}\right\} \in \mathcal{S}_{1} \times \mathcal{S}_{1} \times \mathcal{S}_{2}$.

ii) For brevity, let us not mention again the fact that the inequalities hold for all $\left\{\beta_{k}, \beta_{k-1}, \hat{\beta}_{k}\right\} \in$ $\mathcal{S}_{1} \times \mathcal{S}_{1} \times \mathcal{S}_{2}$. Taking the expected value of (20), assuming null initial conditions $\left(V_{0}=0\right)$, adding the result from $k=0$ to $K-1$, dividing it by $K$ and taking the limit when $K$ tends to infinity we get (19) after considering that $\mathbf{E}\left\{V_{K+1}\right\}>0,\|w\|_{\mathrm{RMS}}^{2} \leq w_{\mathrm{rms}}^{2},\|v\|_{\mathrm{RMS}}^{2} \leq v_{\mathrm{rms}}^{2}$ and $\lim _{K \rightarrow \infty} \frac{1}{K} \sum_{k=0}^{K-1}\left(\mathbf{E}\left\{V_{k}\right\}-V_{k}\right)=0$.

The previous theorem addresses the stability and boundedness of the state estimation error for any possible variation of $\beta_{k}$ and $\hat{\beta}_{k}$ (and thus of $\tilde{\beta}_{k}=\bar{\beta}-\hat{\beta}_{k}$ ). However, for optimization purposes, in this paper we intend to focus on the state estimation performances for each of the different values of the PAR during its steady state. In this aim, we could consider a deterministic approach where no knowledge about the PAR estimation error distribution in its domain (i.e., $\hat{\beta}_{k} \in \mathcal{S}_{2}$ ) is available but, this would lead to conservative results. Note that until now we have just considered that $\tilde{\beta}_{k} \in[\bar{\beta}-1, \bar{\beta}]$, which is the same that $\hat{\beta}_{k} \in \mathcal{S}_{2}$, with probability 1 . However, since $\hat{\beta}_{k}$ comes from filter (5), it would be reasonable to believe that $\hat{\beta}_{k}$ lies with a certain probability in a subset $\mathcal{S}_{3}$ of $\mathcal{S}_{2}$. A first approach to characterize $\mathcal{S}_{3}$ would be to use the probability density function (PDF) of the PAR estimation error. However, obtaining in practical applications this PDF (which makes possible to get the probability $\operatorname{Pr}\left\{\hat{\beta}_{k} \in \mathcal{S}_{3}\right\}$ ) is not an obvious problem. Thanks to the statistical characteristics of the PAR estimation error in steady state obtained in (7), we can instead employ Markov's inequality ${ }^{\S}$, at the expense of introducing some conservativeness, to define a set $\mathcal{S}_{3}$ that imposes a confidence level of $\hat{\beta}_{k} \notin \mathcal{S}_{3}$ lower than $\phi$. The next corollary follows from the previous theorem by applying this idea.

\section{Corollary 4.1}

Consider a given constant PAR $\beta_{k}=\bar{\beta}$ and assume that the PAR estimation algorithm fulfils (7). For given value of $0<a \lesssim 1$ and $0 \lesssim \phi<1$, if there exist positive definite matrices $Q^{o} \in \mathbb{R}^{n \times n}$, and positive scalars $\gamma_{w}^{o, i} \in \mathbb{R}, \gamma_{v}^{o, i} \in \mathbb{R}, \gamma_{w}^{o, o} \in \mathbb{R}$ and $\gamma_{v}^{o, o} \in \mathbb{R}$ fulfilling a modified version of (16) such as

$$
\begin{array}{ll}
M^{o, i}=\left.M^{o}\right|_{\beta_{k}=\beta_{k-1}=\bar{\beta}, P^{o}\left(\beta_{k}\right)=Q^{o}, \gamma_{w}^{o}=\gamma_{w}^{o, i}, \gamma_{v}^{o}=\gamma_{v}^{o, i}} \succ 0, & \forall \hat{\beta}_{k} \in \mathcal{S}_{2}, \hat{\beta}_{k} \in \mathcal{S}_{3}, \\
M^{o, o}=\left.M^{o}\right|_{\beta_{k}=\beta_{k-1}=\bar{\beta}, P^{o}\left(\beta_{k}\right)=Q^{o}, \gamma_{w}^{o}=\gamma_{w}^{o o o}, \gamma_{v}^{o}=\gamma_{v}^{o, o}} \succ 0, & \forall \hat{\beta}_{k} \in \mathcal{S}_{2}, \hat{\beta}_{k} \notin \mathcal{S}_{3},
\end{array}
$$

\footnotetext{
$\S$ If $z$ is a positive random variable and $c>0$, then $\operatorname{Pr}\{z>c\} \leq \frac{\mathbf{E}\{z\}}{c}$.
} 
with

$$
\mathcal{S}_{3}=\left\{\hat{\beta}_{k}: 0 \leq\left(\bar{\beta}-\hat{\beta}_{k}\right)^{2} \leq \frac{1-a}{2 \phi(1+a)} \bar{\beta}(1-\bar{\beta}), \forall k\right\}
$$

then, the statements of Theorem 4.1 apply leading to a state estimation error (under zero initial conditions) bounded by

$$
\mathbf{E}\left\{\|\tilde{x}\|_{\mathrm{RMS}}^{2}\right\}<\gamma_{w}^{o, i} w_{\mathrm{rms}}^{2}+\gamma_{v}^{o, i} v_{\mathrm{rms}}^{2}+\phi\left(\gamma_{w}^{o, o} w_{\mathrm{rms}}^{2}+\gamma_{v}^{o, o} v_{\mathrm{rms}}^{2}\right) .
$$

Proof

Defining the Lyapunov function as $\mathcal{V}_{k}=\mathcal{V}\left(\tilde{x}_{k}\right)=\tilde{x}_{k}^{T} Q^{o} \tilde{x}_{k}$, and by following similar steps as in the proof of Theorem 4.1, constraint (21) and (22) lead respectively to

$$
\begin{aligned}
& \mathbf{E}\left\{\|\tilde{x}\|_{\mathrm{RMS}}^{2} \mid \hat{\beta}_{k} \in \mathcal{S}_{2}, \hat{\beta}_{k} \in \mathcal{S}_{3}\right\}<\gamma_{w}^{o, i} w_{\mathrm{rms}}^{2}+\gamma_{v}^{o, i} v_{\mathrm{rms}}^{2}, \\
& \mathbf{E}\left\{\|\tilde{x}\|_{\mathrm{RMS}}^{2} \mid \hat{\beta}_{k} \in \mathcal{S}_{2}, \hat{\beta}_{k} \notin \mathcal{S}_{3}\right\}<\gamma_{w}^{o, o} w_{\mathrm{rms}}^{2}+\gamma_{v}^{o, o} v_{\mathrm{rms}}^{2} .
\end{aligned}
$$

Using the law of total probability we get ${ }^{\top}$ :

$$
\mathbf{E}\left\{\|\tilde{x}\|_{\mathrm{RMS}}^{2}\right\}<\operatorname{Pr}\left\{\hat{\beta}_{k} \in \mathcal{S}_{3}\right\}\left(\gamma_{w}^{o, i} w_{\mathrm{rms}}^{2}+\gamma_{v}^{o, i} v_{\mathrm{rms}}^{2}\right)+\operatorname{Pr}\left\{\hat{\beta}_{k} \notin \mathcal{S}_{3}\right\}\left(\gamma_{w}^{o, o} w_{\mathrm{rms}}^{2}+\gamma_{v}^{o, o} v_{\mathrm{rms}}^{2}\right) .
$$

From (7) and employing Markov's inequality we obtain that

$$
\operatorname{Pr}\left\{\left(\bar{\beta}-\hat{\beta}_{k}\right)^{2}>\frac{1-a}{2 \phi(1+a)} \bar{\beta}(1-\bar{\beta})\right\} \leq \phi .
$$

From the above expression, $\hat{\beta}_{k}$ (and thus the related PAR estimation error $\left.\left(\bar{\beta}-\hat{\beta}_{k}\right)^{2}\right)$ is in $\mathcal{S}_{3}$ with a probability higher than $1-\phi$. Therefore, we have that $0<\operatorname{Pr}\left\{\hat{\beta}_{k} \notin \mathcal{S}_{3}\right\}<\phi$ and $1-\phi<\operatorname{Pr}\left\{\hat{\beta}_{k} \in\right.$ $\left.\mathcal{S}_{3}\right\} \leq 1$ which allows to find an upper bound of (26) as shown in (24).

\section{Remark 4.1}

Choosing $\phi$ near to 0 enlarges $\mathcal{S}_{3}$ and thus, decreases the probability of $\hat{\beta}_{k}$ being outside of it. In fact, if for a given $\bar{\beta}$ we set $\phi$ such that

$$
\phi<\frac{1-a}{2(1+a)} \min \left\{\frac{1-\bar{\beta}}{\bar{\beta}}, \frac{\bar{\beta}}{1-\bar{\beta}}\right\}
$$

then, $\mathcal{S}_{3}$ becomes redundant since it contains values that exceed the admissible ones given by $\mathcal{S}_{2}$, which means that $\operatorname{Pr}\left\{\hat{\beta}_{k} \in \mathcal{S}_{3}\right\}=1$. On the other hand, choosing $\phi$ near to 1 leads to a small set $\mathcal{S}_{3}$ increasing the probability of the PAR estimation being outside of it. In both of these extreme cases, the resulting state estimation performance is similar to the one we would obtain in a deterministic approach (without any knowledge of the probability distribution of $\hat{\beta}_{k}$ ).

\subsection{Optimization-based observer design}

Addressing at the same time the fulfilment of the constraints from Theorem 4.1 and Corollary 4.1 while minimizing (24) will lead to the observer with gain $L\left(\hat{\beta}_{k}\right)$ that minimizes the upper bound of $\mathbf{E}\left\{\|\tilde{x}\|_{\mathrm{RMS}}^{2}\right\}$ during a given constant PAR $\bar{\beta}$ (see Corollary 4.1) and provides a bounded state estimation error in the general PAR behaviour scenario (see Theorem 4.1). This optimization problem just deals with the case when there is only one steady state PAR. However, in this work we aim to design an estimator capable of adapting its performance to whatever PAR value the network is stabilized in, while assuring robustness against PAR variations and estimation errors.

\footnotetext{
IThese probabilities are equivalent to $\operatorname{Pr}\left\{\hat{\beta}_{k} \in \mathcal{S}_{2}, \hat{\beta}_{k} \in \mathcal{S}_{3}\right\}$ and $\operatorname{Pr}\left\{\hat{\beta}_{k} \in \mathcal{S}_{2}, \hat{\beta}_{k} \notin \mathcal{S}_{3}\right\}$ since $\hat{\beta}_{k}$ is always in $\mathcal{S}_{2}$. As we shall see later, condition $\hat{\beta}_{k} \in \mathcal{S}_{2}$ is added in case the values of $\hat{\beta}_{k}$ in $\hat{\beta}_{k} \in \mathcal{S}_{3}$ exceed the feasible ones given by $\hat{\beta}_{k} \in \mathcal{S}_{2}$.
} 
To reach this goal, we should first modify Corollary 4.1 to explicitly include dependencies of the design result on $\bar{\beta}$ by replacing $Q^{o}, \gamma_{w}^{o, i}, \gamma_{v}^{o, i}, \gamma_{w}^{o, o}$ and $\gamma_{v}^{o, o}$ by $Q^{o}(\bar{\beta}), \gamma_{w}^{o, i}(\bar{\beta}), \gamma_{v}^{o, i}(\bar{\beta}), \gamma_{w}^{o, o}(\bar{\beta})$ and $\gamma_{v}^{o, o}(\bar{\beta})$. After making these changes, we denote the new $M^{o, i}$ and $M^{o, o}$ (see (21) and (22)) by $M^{o, i}(\bar{\beta})$ and $M^{o, o}(\bar{\beta})$. Note that now $\mathcal{S}_{3}$ also depends on $\bar{\beta}$. With that, we present next the proposed parametric optimization-based observer design problem leading to an observer fulfilling statements of Corollary 4.1 for each possible PAR occurrence.

Observer design problem

For given values of $0<a \lesssim 1$ and $0 \lesssim \phi<1$, solve optimization problem

$$
\begin{aligned}
J^{o}(\bar{\beta})=\min & \gamma_{w}^{o, i}(\bar{\beta}) w_{\mathrm{rms}}^{2}+\gamma_{v}^{o, i}(\bar{\beta}) v_{\mathrm{rms}}^{2}+\phi\left(\gamma_{w}^{o, o}(\bar{\beta}) w_{\mathrm{rms}}^{2}+\gamma_{v}^{o, o}(\bar{\beta}) v_{\mathrm{rms}}^{2}\right) \\
\text { s.t. } \quad & M^{o} \succ 0, \quad \forall\left\{\beta_{k}, \beta_{k-1}, \hat{\beta}_{k}\right\} \in \mathcal{S}_{1} \times \mathcal{S}_{1} \times \mathcal{S}_{2}, \\
& M^{o, i}(\bar{\beta}) \succ 0, \quad \forall \hat{\beta}_{k} \in \mathcal{S}_{2}, \hat{\beta}_{k} \in \mathcal{S}_{3}(\bar{\beta}), \\
& M^{o, o}(\bar{\beta}) \succ 0, \quad \forall \hat{\beta}_{k} \in \mathcal{S}_{2}, \hat{\beta}_{k} \notin \mathcal{S}_{3}(\bar{\beta})
\end{aligned}
$$

over $\bar{\beta} \in\left[\beta_{\min }, 1\right]$.

\section{Remark 4.2}

The convergence speed of the PAR estimation algorithm (5), and thus the transient time lasted until Corollary 4.1 is applicable, when the PAR is on steady state after a given change, is given by parameter $a$. As mentioned in Remark 2.1, the convergence time to $\bar{\beta}$ after a step change can be characterized by $\log (0.02) / \log (a)$. Then, choosing lower values of $a$ reduces the transient time where Theorem 4.1 holds at the expense of enlarging $\mathcal{S}_{3}$ (higher PAR estimation errors and thus worse capability of adaptation in steady state.).

We shall discuss the numerical solvability of the observer design problem (28) in Section 6.

\section{CONTROLLER DESIGN}

In this section we study the design of the controller with law $K\left(\hat{\beta}_{k}\right)$ and $F\left(\hat{\beta}_{k}\right)$. Let us first restate the resulting closed loop dynamics from (13a) using vector $z_{k}=\left[\begin{array}{ll}x_{k}^{T} & u_{k-1}^{T}\end{array}\right]^{T}$ as

$$
z_{k+1}=\left(\mathcal{A}\left(\delta_{k}\right)+\mathcal{B}_{u}\left(\delta_{k}\right) \mathcal{K}\left(\hat{\beta}_{k}\right)\right) z_{k}+\mathcal{B}_{w} w_{k}-\mathcal{B}_{u}\left(\delta_{k}\right) K\left(\hat{\beta}_{k}\right) \tilde{x}_{k},
$$

with $z_{k} \in \mathbb{R}^{n_{z}}, n_{z}=n+n_{u}$ and

$$
\mathcal{A}\left(\delta_{k}\right)=\left[\begin{array}{cc}
A & \left(1-\delta_{k}\right) \mu B_{u} \\
0 & \left(1-\delta_{k}\right) \mu I
\end{array}\right], \mathcal{B}_{u}\left(\delta_{k}\right)=\left[\begin{array}{c}
\delta_{k} B_{u} \\
\delta_{k} I
\end{array}\right], \mathcal{K}\left(\hat{\beta}_{k}\right)=\left[\begin{array}{cc}
K\left(\hat{\beta}_{k}\right) & F\left(\hat{\beta}_{k}\right)
\end{array}\right], \mathcal{B}_{w}=\left[\begin{array}{c}
B_{w} \\
0
\end{array}\right] .
$$

Note that in (29), the controller gain matrices are aggregated in $\mathcal{K}\left(\hat{\beta}_{k}\right)$. In order to aggregate the control gain $K\left(\hat{\beta}_{k}\right)$ affecting the state estimation error with $F\left(\hat{\beta}_{k}\right)$ into $\mathcal{K}\left(\hat{\beta}_{k}\right)$, we rewrite (29) such as

$$
z_{k+1}=\left(\mathcal{A}\left(\delta_{k}\right)+\mathcal{B}_{u}\left(\delta_{k}\right) \mathcal{K}\left(\hat{\beta}_{k}\right)\right) z_{k}+\mathcal{B}_{w} w_{k}-\mathcal{B}_{u}\left(\delta_{k}\right) \mathcal{K}\left(\hat{\beta}_{k}\right) \xi_{k}
$$

where $\xi_{k}=\left[\begin{array}{ll}\tilde{x}_{k}^{T} & 0\end{array}\right]^{T}$.

Bearing this in mind, we next provide a procedure based on sufficient PDMI conditions to design the observer-based controller with gains $K\left(\hat{\beta}_{k}\right)$ and $F\left(\hat{\beta}_{k}\right)$ fulfilling the design goals stated in Section 3.2. First, the next theorem shows under which conditions the closed loop state $z_{k}$ is bounded subject to a general PAR scenario with any possible PAR estimation error.

Theorem 5.1

Consider the control algorithm (12) applied to system (1), where $\hat{x}_{k}$ comes from algorithm (10) with 
the gains designed by (28), and the PAR estimation algorithm (5). For a given positive scalar $\varepsilon \in \mathbb{R}$, $\mu \in[0,1]$ and a matrix $R \in \mathbb{R}^{n_{z} \times n_{z}}$, if there exist positive definite matrices $P^{c}\left(\beta_{k}\right) \in \mathbb{R}^{n_{z} \times n_{z}}$, full matrices $G^{c}\left(\hat{\beta}_{k}\right) \in \mathbb{R}^{n_{z} \times n_{z}}$ and $X^{c}\left(\hat{\beta}_{k}\right) \in \mathbb{R}^{n_{u} \times n_{z}}$, and positive scalars $\gamma_{w}^{c} \in \mathbb{R}, \gamma_{v}^{c} \in \mathbb{R}$ and $\gamma_{0}^{c} \in \mathbb{R}$, fulfilling

$$
\begin{aligned}
M^{c} & =\left[\begin{array}{ccc}
M_{11}^{c} & M_{12}^{c} & M_{13}^{c} \\
\star & M_{22}^{c} & M_{23}^{c} \\
\star & \star & M_{33}^{c}
\end{array}\right] \succ 0, \quad \forall\left\{\beta_{k}, \beta_{k-1}, \hat{\beta}_{k}\right\} \in \mathcal{S}_{1} \times \mathcal{S}_{1} \times \mathcal{S}_{2}, \\
N^{c} & =G^{c}\left(\hat{\beta}_{k}\right)+G^{c}\left(\hat{\beta}_{k}\right)^{T}-(1+\varepsilon) I \succ 0, \quad \forall \hat{\beta}_{k} \in \mathcal{S}_{2},
\end{aligned}
$$

with

$$
\begin{aligned}
& M_{11}^{c}=\beta_{k} P^{c}\left(\beta_{k}\right) \oplus\left(1-\beta_{k}\right) P^{c}\left(\beta_{k}\right), \quad M_{22}^{c}=G^{c}\left(\hat{\beta}_{k}\right)+G^{c}\left(\hat{\beta}_{k}\right)^{T}-P^{c}\left(\beta_{k-1}\right), \\
& M_{12}^{c}=\left[\begin{array}{lll}
\beta_{k}\left(\mathcal{A}(1) G^{c}\left(\hat{\beta}_{k}\right)+\mathcal{B}_{u}(1) X^{c}\left(\hat{\beta}_{k}\right)\right)^{T} & \left(1-\beta_{k}\right)\left(\mathcal{A}(0) G^{c}\left(\hat{\beta}_{k}\right)\right)^{T}
\end{array}\right]^{T}, \\
& M_{13}^{c}=\left[\begin{array}{ccc}
\beta_{k} \mathcal{B}_{w} & \beta_{k} \mathcal{B}_{u}(1) X^{c}\left(\hat{\beta}_{k}\right) & 0 \\
\left(1-\beta_{k}\right) \mathcal{B}_{w} & 0 & 0
\end{array}\right], \\
& M_{23}^{c}=\left[\begin{array}{lll}
0 & 0 & G^{c}\left(\hat{\beta}_{k}\right)^{T} R^{T}
\end{array}\right], \quad M_{33}^{c}=\left(\gamma_{w}^{c} I\right) \oplus\left(\gamma_{v}^{c} \varepsilon I\right) \oplus\left(\gamma_{0}^{c} \varepsilon I\right) \oplus I,
\end{aligned}
$$

then, defining the controller gain matrices as $\mathcal{K}\left(\hat{\beta}_{k}\right)=X^{c}\left(\hat{\beta}_{k}\right) G^{c}\left(\hat{\beta}_{k}\right)^{-1}$ : i) in the absence of disturbances and estimation errors the average closed loop state $z_{k}$ in (29) converges to zero, ii) under zero initial conditions, the system states and control inputs are bounded through $z_{k}$ by

$$
\mathbf{E}\left\{\|R \tilde{z}\|_{\mathrm{RMS}}^{2}\right\}<\gamma_{w}^{c} \bar{w}_{\mathrm{rms}}^{2}+\gamma_{v}^{c} \tilde{x}_{\mathrm{rms}}^{2}
$$

with $\tilde{x}_{\mathrm{rms}}=\gamma_{w}^{o} \bar{w}_{\mathrm{rms}}^{2}+\gamma_{v}^{o} \bar{v}_{\mathrm{rms}}^{2}$, see (19).

\section{Proof}

Using the fact that $\left(G^{c}\left(\hat{\beta}_{k}\right)-I\right)^{T}\left(G^{c}\left(\hat{\beta}_{k}\right)-I\right) \succeq 0$, if (33) holds, then $G^{c}\left(\hat{\beta}_{k}\right)^{T} G^{c}\left(\hat{\beta}_{k}\right) \succ \varepsilon I$. Thus, if (32) holds, the resulting PDMI from replacing $\varepsilon I$ by $G^{c}\left(\hat{\beta}_{k}\right)^{T} G^{c}\left(\hat{\beta}_{k}\right)$ in (32) also holds. Then, if we define the Lyapunov function as $V_{k}=V\left(z_{k}, \beta_{k-1}\right)=\tilde{x}_{k}^{T} P^{c}\left(\beta_{k-1}\right)^{-1} \tilde{x}_{k}$, applying a congruence transformation on the above modification over (32) with matrix $I \oplus I \oplus\left(G^{c}\left(\hat{\beta}_{k}\right)^{T}\right)^{-1} \oplus I \oplus$ $\left(G^{c}\left(\hat{\beta}_{k}\right)^{T}\right)^{-1} \oplus\left(G^{c}\left(\hat{\beta}_{k}\right)^{T}\right)^{-1} \oplus I$, taking Schur's complements and premultiplying the result by $\left[\begin{array}{lll}z_{k}^{T} & w_{k-1}^{T} & \xi_{k}^{T}\end{array}\right]$ (with $\xi_{k}$ as defined in (31)) and postmultiplying by its transpose leads to

$$
\mathbf{E}\left\{V_{k+1}\right\}-V_{k}+z_{k}^{T} R^{T} R z_{k}-\gamma_{w}^{c} w_{k}^{T} w_{k}-\gamma_{v}^{c} \tilde{x}_{k}^{T} \tilde{x}_{k}<0
$$

for all $\left\{\beta_{k}, \beta_{k-1}, \hat{\beta}_{k}\right\} \in \mathcal{S}_{1} \times \mathcal{S}_{1} \times \mathcal{S}_{2}$, where we have taken into account that $\mathcal{B}_{u}(0)=0$ and $\xi_{k}=\left[\begin{array}{ll}\tilde{x}_{k}^{T} & 0\end{array}\right]^{T}$. From (36) and following similar arguments as in the proof of Theorem 4.1, one can prove the assertions of the current theorem.

\section{Remark 5.1}

Parameters $\varepsilon$ and $\mu$ can be set as line search parameters for conservatism reduction.

\section{Remark 5.2}

Let us highlight some structural modifications and constraints used in Theorem 5.1 to convexify the design of observer-based controllers, which is usually a nonconvex problem [15]. First, the use of gain matrix $F\left(\hat{\beta}_{k}\right)$ compensating the previous control input in (12) together with the reformulation of the closed loop dynamics (29) performed in (31) through $\xi_{k}$ allows preserving the product $X\left(\hat{\beta}_{k}\right)=\mathcal{K}\left(\hat{\beta}_{k}\right) G\left(\hat{\beta}_{k}\right)$ all over matrix (32). Second, in the aim of [15] but employing the slack variable $G\left(\hat{\beta}_{k}\right)$ (which decouples the product between the Lyapunov and system matrices), condition (33) allows us to replace the product of $G\left(\hat{\beta}_{k}\right)^{T} G\left(\hat{\beta}_{k}\right)$ on the diagonal elements of (32) by some given positive value $\varepsilon$ that fulfils (33). 
Second, following the proposed design procedure in Section 3.2 and in the aim of the reasoning of the observer design procedure (see Section 4), the next corollary (which follows form Theorem 5.1) describes the control performances for each of the different values of the PAR during its steady state for any associated $\tilde{\beta}_{k}$.

Corollary 5.1

Consider a given constant PAR $\beta_{k}=\bar{\beta}$ and assume that the PAR estimation algorithm fulfils (7). For given value of $0<a \lesssim 1$ and $0 \lesssim \phi<1$, if there exist positive definite matrices $Q^{c} \in \mathbb{R}^{n \times n}$, and positive scalars $\gamma_{w}^{c, i} \in \mathbb{R}, \gamma_{v}^{c, i} \in \mathbb{R}, \gamma_{w}^{c, o} \in \mathbb{R}, \gamma_{v}^{c, o} \in \mathbb{R}, \gamma_{0}^{c, o} \in \mathbb{R}$ and,$\gamma_{0}^{c, o} \in \mathbb{R}$ fulfilling (33) and a modified version of (32) such as

$$
\begin{array}{ll}
M^{c, i}=\left.M^{c}\right|_{\beta_{k}=\beta_{k-1}=\bar{\beta}, P^{c}\left(\beta_{k}\right)=Q^{c}, \gamma_{w}^{c}=\gamma_{w}^{c, i}, \gamma_{v}^{c}=\gamma_{v}^{c, i} \gamma_{0}^{c}=\gamma_{0}^{c, i}} \succ 0, \quad \forall \hat{\beta}_{k} \in \mathcal{S}_{2}, \hat{\beta}_{k} \in \mathcal{S}_{3}, \\
M^{c, o}=\left.M^{c}\right|_{\beta_{k}=\beta_{k-1}=\bar{\beta}, P^{c}\left(\beta_{k}\right)=Q^{c}, \gamma_{w}^{c}=\gamma_{w}^{c, o}, \gamma_{v}^{c}=\gamma_{v}^{c, o} \gamma_{0}^{c}=\gamma_{0}^{c, o} \succ 0,} \succ \hat{\beta}_{k} \in \mathcal{S}_{2}, \hat{\beta}_{k} \notin \mathcal{S}_{3},
\end{array}
$$

then, the statements of Theorem 5.1 apply leading to a closed loop state (under zero initial conditions) bounded by

$$
\mathbf{E}\left\{\|R z\|_{\mathrm{RMS}}^{2}\right\}<\gamma_{w}^{c, i} w_{\mathrm{rms}}^{2}+\gamma_{v}^{c, i} J^{o, i}+\phi\left(\gamma_{w}^{c, o} w_{\mathrm{rms}}^{2}+\gamma_{v}^{c, o} J^{o, o}\right)
$$

with $J^{o, i}=\gamma_{w}^{o, i} w_{\mathrm{rms}}^{2}+\gamma_{v}^{o, i} v_{\mathrm{rms}}^{2}$ and $J^{o, o}=\gamma_{w}^{o, o} w_{\mathrm{rms}}^{2}+\gamma_{v}^{o, o} v_{\mathrm{rms}}^{2}$ being the result of problem (28) for the given $\bar{\beta}$.

Proof

Following similar arguments than in the proof of Corollary 4.1 together with the one of Theorem 5.1, we can obtain (39).

\subsection{Optimization-based controller design}

Finally, as in the observer design section, conditions of Theorem 5.1 and Corollary 5.1 can be joined into an optimization problem to minimize the closed loop state RMS norm for a given constant PAR $\bar{\beta}$ while providing robustness against PAR variations and estimation error (both on the PAR and system states). Following similar arguments as in the last section, we next present the parametric optimization-based design problem for all possible $\bar{\beta} \in\left[\beta_{\min }, 1\right]$. Note that we have replaced $Q^{c}$, $\gamma_{w}^{c, i}, \gamma_{v}^{c, i}, \gamma_{0}^{c, i}, \gamma_{w}^{c, o}, \gamma_{v}^{c, o}$ and $\gamma_{0}^{c, o}$ by $Q^{c}(\bar{\beta}), \gamma_{w}^{c, i}(\bar{\beta}), \gamma_{v}^{c, i}(\bar{\beta}), \gamma_{0}^{c, i}(\bar{\beta}), \gamma_{w}^{c, o}(\bar{\beta}), \gamma_{v}^{c, o}(\bar{\beta})$ and $\gamma_{0}^{c, o}(\bar{\beta})$. We denote the resulting $M^{c, i}$ and $M^{c, o}$ (see (37) and (38) ) by $M^{c, i}(\bar{\beta})$ and $M^{c, o}(\bar{\beta})$.

\section{Controller design problem}

For a given positive scalar $\varepsilon \in \mathbb{R}$, real value parameters $\mu \in[0,1], 0<a \lesssim 1$ and $0 \lesssim \phi<1$, and a matrix $R \in \mathbb{R}^{n_{z} \times n_{z}}$, solve optimization problem

$$
\begin{aligned}
J^{c}(\bar{\beta})=\min & \gamma_{w}^{c, i}(\bar{\beta}) w_{\mathrm{rms}}^{2}+\gamma_{v}^{c, i}(\bar{\beta}) J^{o, i}(\bar{\beta})+\phi\left(\gamma_{w}^{c, o}(\bar{\beta}) w_{\mathrm{rms}}^{2}+\gamma_{v}^{c, o}(\bar{\beta}) J^{o, o}(\bar{\beta})\right) \\
\text { s.t. } \quad & M^{c} \succ 0, \quad \forall\left\{\beta_{k}, \beta_{k-1}, \hat{\beta}_{k}\right\} \in \mathcal{S}_{1} \times \mathcal{S}_{1} \times \mathcal{S}_{2}, \\
& M^{c, i}(\bar{\beta}) \succ 0, \quad \forall \hat{\beta}_{k} \in \mathcal{S}_{2}, \hat{\beta}_{k} \in \mathcal{S}_{3}(\bar{\beta}), \\
& M^{c, o}(\bar{\beta}) \succ 0, \quad \forall \hat{\beta}_{k} \in \mathcal{S}_{2}, \hat{\beta}_{k} \notin \mathcal{S}_{3}(\bar{\beta}), \\
& N^{c} \succ 0 \quad \forall \hat{\beta}_{k} \in \mathcal{S}_{2},
\end{aligned}
$$

over $\bar{\beta} \in\left[\beta_{\min }, 1\right]$.

\section{Remark 5.3}

Matrix $R$ in (40) is a weighting factor that balances between the convergence speed to the origin of the states $x_{k}$ and the magnitude of the control inputs $u_{k}$. By choosing $R$ as a positive definite block diagonal matrix one obtains the classical tuning parameters of optimal control [22]. In the aim of simplicity, we propose to select $R=\sqrt{C^{T} C} \oplus \sqrt{\lambda} I$. Then, the optimization problem tries to minimize $\|y\|_{\mathrm{RMS}}^{2}+\lambda\|u\|_{\mathrm{RMS}}^{2}$ where $\lambda$ is a tuning parameter.

We shall discuss the numerical solvability of the controller design problem (40) in the next section. 


\section{NUMERICAL ISSUES}

Solving optimization problems (28) and (40) implies verifying the PDMI conditions over the entire parameter space of the PAR, which leads to an infinite-dimensional problem. To offer a wider solution, we propose to impose polynomial dependences on $\left\{\beta_{k}, \beta_{k-1}, \hat{\beta}_{k}, \bar{\beta}\right\}$ of fixed degree on the parametrized matrices and scalar functions. With that, SOS decompositions or homogeneous polynomial techniques can be used to obtain a computationally tractable procedure [21]. Usually, homogeneous polynomial are employed when the parameters are constrained in the simplex. However, in the analysed case, transforming $\hat{\beta}_{k} \in \mathcal{S}_{3}(\bar{\beta})$ into a restriction over a simplex domain is far from obvious. For this reason, we propose to address the solvability of (28) and (40) by using a SOS decomposition that gives sufficient conditions on the positiveness of the PDMIs. Details on how to perform this SOS-based approach to deal with the feasibility problem can be found in $[21,27]$.

\section{Remark 6.1}

In the case that the system is unstable but detectable, the authors in [28] showed that it is necessary that the PAR fulfils $\beta_{k} \geq 1-\frac{1}{\rho(A)^{2}}$ (being $\rho(A)=\max _{i}\left(\left|\lambda_{i}(A)\right|\right)$ and $\lambda_{i}(A)$ the eigenvalues of matrix $A$ ) to make the existence of a solution for the observer and controller design possible. Thus, we must assume that the PAR satisfies this bound.

Concerning the minimization of the parametric cost index in (28) and (40), one possible way to address it could be to perform a minimization of an upper bound of $J^{\chi}(\bar{\beta})$, with $\chi \in\{o, c\}$, for all $\bar{\beta} \in\left[\beta_{\text {min }}, 1\right]$. This would lead to the design of the worst case observer and controller (robust solution). In the aim of achieving less conservative results and in order to cope with the parametrized optimization over $\bar{\beta}$, we propose, as an alternative, to introduce a weighting function $g(\bar{\beta})$ such that

$$
\bar{J}^{\chi}=\int_{\mathcal{S}_{4}} g(\bar{\beta}) J^{\chi}(\bar{\beta}) \mathrm{d} \bar{\beta}
$$

with $\mathcal{S}_{4}=\left\{\bar{\beta}: \beta_{\min } \leq \bar{\beta} \leq 1\right\}$ and $\chi \in\{o, c\}$. Then, minimizing $\bar{J}^{\chi}$ leads to the gain-scheduled observer-based controller that minimizes the RMS norm of the state estimation error and the RMS norm of the closed loop states under the weighting function $g(\bar{\beta})$.

\section{Remark 6.2}

The weighting function $g(\bar{\beta})$ aims to involve the different values of the objective function $J^{\chi}(\bar{\beta})$ for each $\bar{\beta}$ into the minimization. A thoughtful choice is setting

$$
g(\bar{\beta})= \begin{cases}\frac{1}{1-\beta_{\min }} & \text { if } \beta_{\min } \leq \bar{\beta} \leq 1 \\ 0 & \text { otherwise. }\end{cases}
$$

With this weighting function, the minimization problem tries to minimize the expected value of $J^{\chi}(\bar{\beta})$ when the PAR is equally likely to be stabilized in any $\bar{\beta} \in\left\{\beta_{\min }, 1\right\}$.

\section{Remark 6.3}

So far we have supposed that $0 \lesssim \phi<1$ was a parameter to be chosen. $\phi$ could be selected according to the time-varying behaviour of the PAR. If the PAR is known to change rarely and we choose parameter $a$ near 1 , as stated in Remark 2.1, the PAR estimation algorithm will give accurate estimation in steady state. Therefore, to include this information in the design procedure we could choose lower values of $\phi$ increasing the probability of being inside $\mathcal{S}_{3}$ without enlarging it much for optimization purposes. On the other hand, if the PAR varies permanently and we tune $a$ accordingly, $\tilde{\beta}_{k}$ will be higher in steady state and then we should set $\phi$ to higher values to narrow $\mathcal{S}_{3}$ so it does not encompass $\mathcal{S}_{2}$. However if no a priori information is available, and as a general approach, we propose to get its value from the optimization problem by performing a line search in $\phi$ such that the value of $\bar{J}^{o}$ (from the observer design) is minimum and then, use the same value in the controller design since it gives the minimum state estimation error. Although optimizing over $\phi$ minimizes $\bar{J}^{o}$, 
and thus, gives a tighten bound on the estimation performance index, it might not lead to the optimal observer-based controller in practice, i.e., the one that obtains the minimum $\|\tilde{x}\|_{\mathrm{RMS}}$ and $\|z\|_{\mathrm{RMS}}$ in implementation.

\section{SIMULATION EXAMPLE}

In this example we aim to show the behaviour of the proposed observer-based controllers. For that purpose, we will first analyse the influence of parameter $a$ and $\phi$ in the PAR estimation algorithm and in the resulting gain-scheduling approach. Then, we will study the behaviour in simulation of different observer-based controllers under sporadic and frequent changes of the PAR and compare the results with the approach in [22].

Let us consider the position control problem of a DC motor with transfer function

$$
G(s)=\frac{5.8}{s(1+0.033 s)(1+0.006 s)} .
$$

Discretizing the system with a sampling period of $T_{s}=10 \mathrm{~ms}$ we obtain the following state-space representation defined by (1):

$$
A=\left[\begin{array}{ccc}
1.927 & -1.067 & 0.279 \\
1 & 0 & 0 \\
0 & 0.5 & 0
\end{array}\right], \quad B_{u}=B_{w}=\left[\begin{array}{c}
0.125 \\
0 \\
0
\end{array}\right], \quad C=\left[\begin{array}{lll}
0.0249 & 0.0641 & 0.0188
\end{array}\right]
$$

We consider an actuator strategy with $\mu=1$ and the state disturbance and measurement noise to have bounded RMS norms given by:

$$
\|w\|_{\mathrm{RMS}}=0.6, \quad\|v\|_{\mathrm{RMS}}=0.01 .
$$

Here, the state disturbance is such as $w_{k}=0.6 \cdot(-1)^{k}$ (representing the worst sampling case of a sinusoidal signal of amplitude 0.6 and frecuency $50 \mathrm{~Hz}$ ), while the measurement noise modelling the accuracy of the position sensor (typically an encoder) is a Gaussian noise of variance $10^{-4}$. We also assume that the network provides a lower bound of the PAR given by $\beta_{\min }=0.3$, which is used to solve the design problems (see Remark 6.1).

Let us first analyse the influence of parameter $a$ in the PAR estimation (see (5)). Figure 2 shows the PAR estimation performance for the cases when $a=0.999$ and $a=0.9$. When $a$ is lower, the estimated PAR is able to follow faster changes of the real PAR (lower transient time where only Theorem 4.1 and Theorem 5.1 apply). In fact, following Remark 2.1, when $a=0.9$, it takes $0.37 \mathrm{sec}$ to track the change in PAR with an average estimation error lower than $2 \%$. This is 100 times faster than using $a=0.999$. However, the obtained PAR estimation error in steady state is much higher when using $a=0.9$.

Second, we examine how the pair $(a, \phi)$ affects the size of set $\mathcal{S}_{3}$, which contains the PAR estimation error in steady state with a probability higher than $1-\phi$ (see (23)). If we fix $\phi=0.01$ (i.e., $\operatorname{Pr}\left\{\hat{\beta}_{k} \in \mathcal{S}_{3}\right\}>0.99$ ) for PAR estimators, in Figure 2 we verify that a lower $a$ leads to a wider range of values in $\mathcal{S}_{3}$, which might lead to more conservative results. Also, note that, as mentioned in Remark 4.1, $\mathcal{S}_{3}$ might contain values of $\hat{\beta}_{k}$ (and thus of $\tilde{\beta}_{k}$ ) that are outside of $\mathcal{S}_{2}$ and therefore are unreachable. In fact, when $\bar{\beta}=0.4$ we find from (27) that $\phi<0.0175$ implies that $\mathcal{S}_{3}$ is redundant. This phenomenon is due to the conservativeness introduced by the use of Markov's inequality.

Let us now study the state observers and controllers resulting from the proposed methods in Section 4 and Section 5 with $\lambda=0.1$ (see Remark 5.3). For this purpose, Figure 3 and Figure 4 display the possible values (shadowed grey region) that take the observer and controller gains, and their respective performances, for the possible combinations of $a \in[0.9,0.999]$ and $\phi \in[0.001,0.2]$ when using polynomials of degree 4 . To ease of representation, we have normalized the values by 

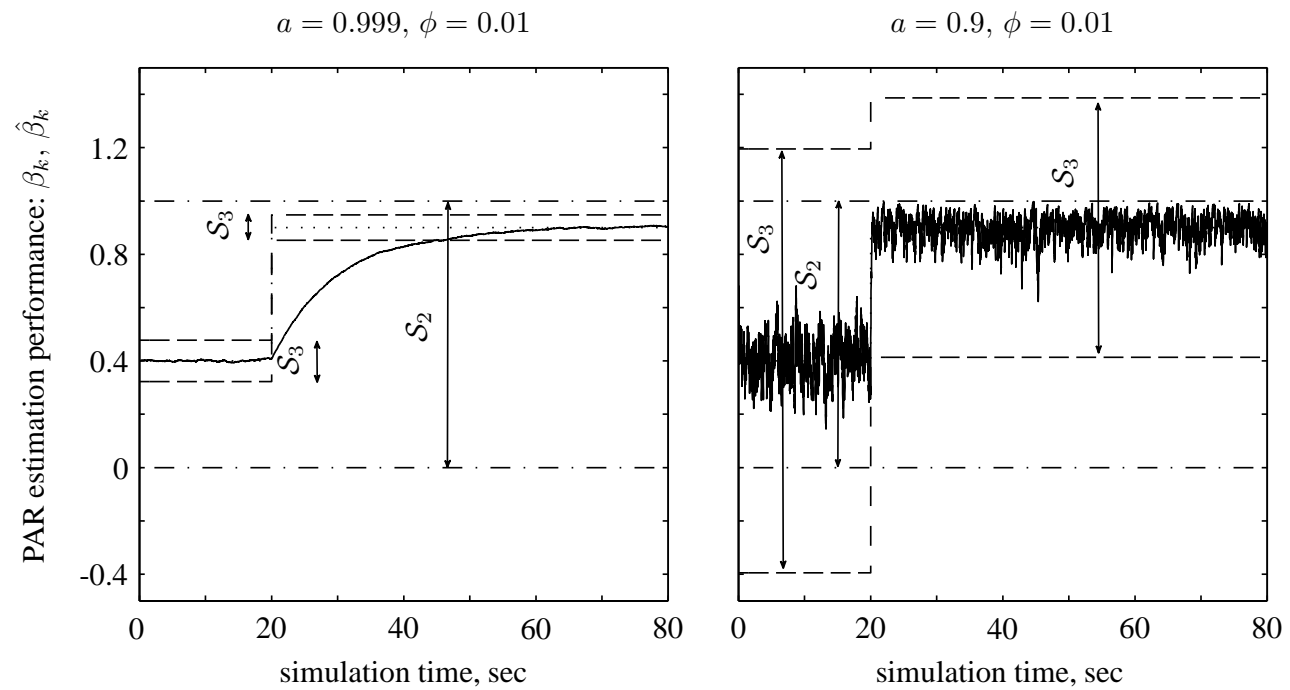

Figure 2. PAR estimation performances of the proposed filter (5) for different values of $a$ and $\phi=0.01$. Analysis of sets $\mathcal{S}_{2}$ and $\mathcal{S}_{3}$.

dividing them by:

$$
\begin{aligned}
& \max \left(\left|L\left(\hat{\beta}_{k}\right)\right|\right)=11.747, \quad \max \left(\left|J^{o}(\bar{\beta})\right|\right)=4.122 \\
& \max \left(\left|\mathcal{K}\left(\hat{\beta}_{k}\right)\right|\right)=0.933, \quad \max \left(\left|J^{c}(\bar{\beta})\right|\right)=3.325
\end{aligned}
$$

For a fixed $\phi$, lower values of a (faster PAR estimation convergence to steady state where Corollary 4.1 and 5.1 hold) make the resulting observer-based controller more robust to PAR estimation errors in steady state (gains are almost independent of $\hat{\beta}_{k}$ ) leading to conservative performances since $\mathcal{S}_{3}$ is larger. On the other hand, higher values of a narrow $\mathcal{S}_{3}$ allowing to get more adaptive solutions with lower performance cost indexes in steady state at the expense of having worse PAR tracking performances (larger transient behaviour where only Theorem 4.1 and 5.1 apply). For a fixed $a$, too low values of $\phi$ result in robust solutions since $\mathcal{S}_{3}$ is larger while too high values of $\phi$ give similar performance because the weighting factor of being outside $\mathcal{S}_{3}$ dominates de optimization problem. Then, intermediate but lower enough values of $\phi$ lead to the adaptive solutions. These figures prove that scheduling the gains with the estimated PAR allows increasing the estimation and controller performances (lower values of $J^{o}(\bar{\beta})$ and $J^{c}(\bar{\beta})$ when the PAR is lower and vice-versa).

Table I. Analysed cases. '*’ shows a value obtained through optimization.

\begin{tabular}{|c|c|c|c|}
\hline Case & $a$ & $\phi$ & Design method \\
\hline C1 & - & - & Theorem 4.1/5.1 \\
C2 & 0.999 & - & Problem $(28) /(40)$ with constant gains \\
C3 & - & - & Problem $(28) /(40)$ without $\mathcal{S}_{3}$ \\
C4 & 0.999 & $0.005^{*}$ & Problem $(28) /(40)$ \\
C5 & 0.999 & $10^{-5}$ & Problem $(28) /(40)$ \\
C6 & 0.999 & 0.2 & Problem $(28) /(40)$ \\
C7 & 0.999 & - & LQG [22] \\
C8 & 0.99 & 0.005 & Problem $(28) /(40)$ \\
C9 & 0.99 & - & LQG $[22]$ \\
\hline
\end{tabular}



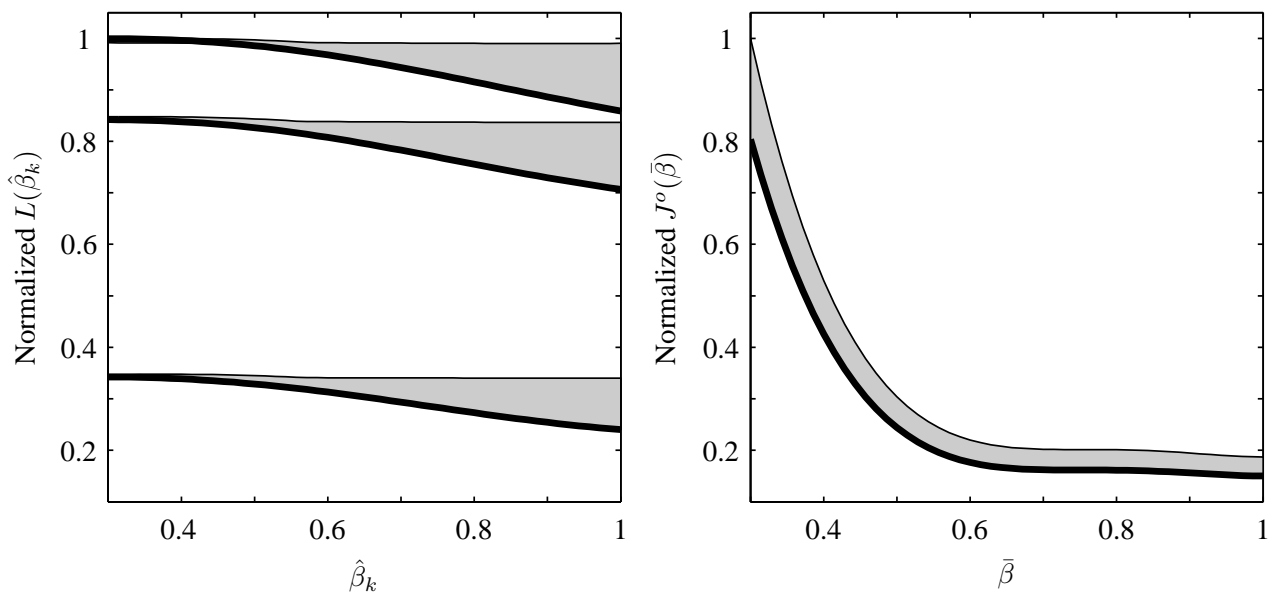

Figure 3. Normalized scheduled observer gain $L\left(\hat{\beta}_{k}\right)$ and estimation performance $J^{o}(\bar{\beta})$. Shadowed zone represents the resulting values for $a \in[0.9,0.999]$ and $\phi \in[0.001,0.2]$. The thickest line stands for case C4.
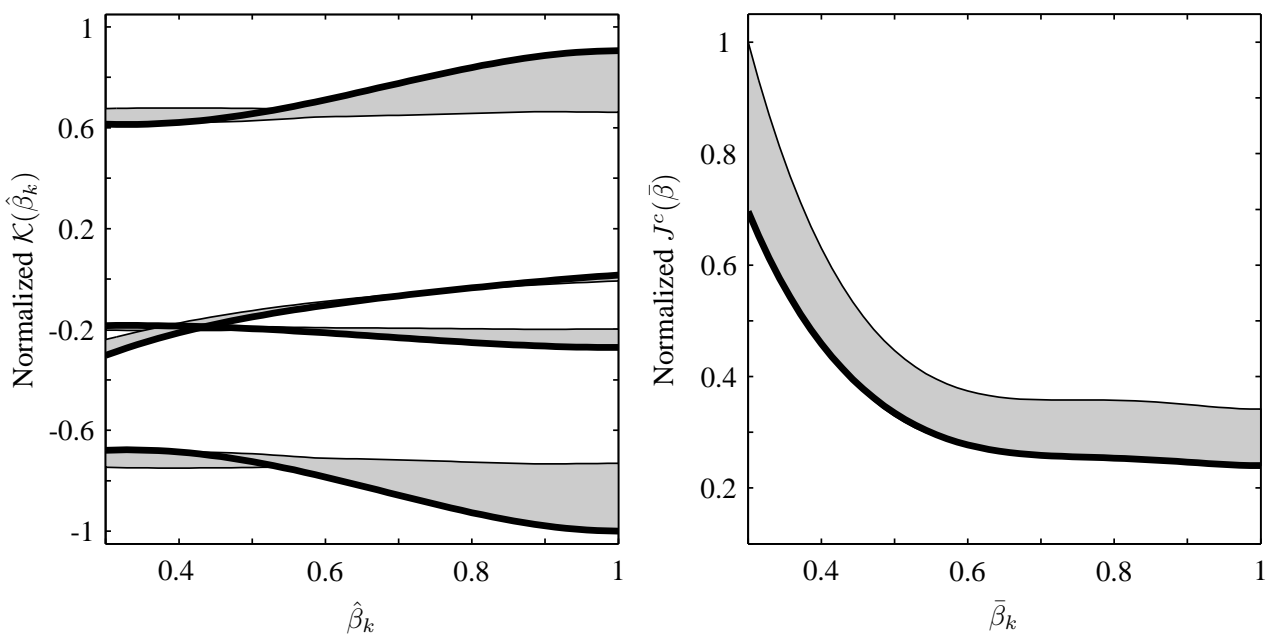

Figure 4. Normalized scheduled controller gain $\mathcal{K}\left(\hat{\beta}_{k}\right)$ and control performance $J^{c}(\bar{\beta})$. Shadowed zone represents the resulting values for $a \in[0.9,0.999]$ and $\phi \in[0.001,0.2]$. The thickest line stands for case C4.

Let us now examine the performances of the observer-based controllers in Table I. C1 only considers the case when the PAR varies at each instant through conditions from Theorem 4.1 and 5.1 while minimizing (19) and (35) respectively. All the design approaches but C2, C7 and C8 have considered polynomial dependences of degree 4 . In C3 we include possible steady state PAR behaviours by adding constraints (21) and (37) with $\hat{\beta}_{k}$ belonging just to $\mathcal{S}_{2}$ and by minimizing the first two terms of (24) and (39). C2, C4, C5, C6 and C8 are designed following problems (28) and (40). However, gains of $\mathrm{C} 2$ are not scheduled with $\hat{\beta}_{k}$ (constant gains). The obtained gains for $\mathrm{C} 2$ are $L=\left[\begin{array}{lll}11 & , 9.709 & 4.189\end{array}\right]^{T}, \mathcal{K}=\left[\begin{array}{llll}-0.293 & 0.271 & -0.082 & -0.339\end{array}\right]^{T}$. Also, we have only performed the line search approach over $\phi$ to minimize the cost index (proposed in Remark 6.3) in C4. Note that using the proposed approach to choose $\phi$ as the one that optimizes $\bar{J}^{o}$ (see Remark 6.3), leads to the best controller index performance $\bar{J}^{c}$ as shown in Figure 4. C5 considers a lower $\phi$ than $\mathrm{C} 4$ while $\mathrm{C} 6$ is designed with a higher one. Instead, C8 implements a PAR estimator with a faster dynamic than $\mathrm{C} 4$. To compare the results of the current paper with existing approaches, 
we have implemented the LQG approach with infinite horizon of [22]. Since the controller design at each instant depends on the unknown $\beta_{k}$, we have used instead the resulting $\hat{\beta}_{k}$ from PAR estimators with $a=0.999$ (C7) and $a=0.99$ (C9).

In order to evaluate the control performances we propose the following index

$$
J_{k}^{c}=\frac{1}{k} \sum_{i=0}^{k-1} x_{i}^{T} x_{i}+\lambda \frac{1}{k} \sum_{i=0}^{k-1} u_{i}^{T} u_{i}
$$

Table II shows the obtained performances in simulation at time $600 \mathrm{sec}$ of the stabilization problem from $x_{0}=\left[\begin{array}{lll}4 & 4 & 4\end{array}\right]^{T}$ under three PAR scenarios: i) $\beta_{k}$ is time-invariant with a value of 0.4 , ii) $\beta_{k}$ changes each 100sec between 0.9 and 0.4 and, iii) $\beta_{k}$ varies following a uniform distribution from 0.3 to 0.95 each $5 \mathrm{sec}$. Thanks to having focused on estimation and control performance when the PAR is on its steady state, we reduce the control performance index $J_{k}^{c}$ up to a $65 \%$ with respect to $\mathrm{C} 1$, where only the general PAR scenario is considered. Scheduling the observer and controller gain also allow to reduce it up to a 55\% with respect to C2 (constant gains). The design approach developed in this work allows us to reduce the control performance index of the LQG approach (C7 and C9) up to a $25 \%$ because: i) the considered state disturbance is not Gaussian nor uncorrelated (but with a zero mean), ii) our approach takes into account the PAR estimation error. Note that there is no big difference between the performances of $\mathrm{C} 7$ and $\mathrm{C} 9$.

Let us now compare the results of $\mathrm{C} 4, \mathrm{C} 5, \mathrm{C} 6$ and $\mathrm{C} 8$. C5 performs similar as the deterministic case $\mathrm{C} 3$ since the chosen $\phi$ is lower than stated in Remark 4.1 and thus, $\mathcal{S}_{3}$ becomes redundant for the considered PARs. In general, C4 gives better performance since for this case $\phi$ was a design parameter too. However there are a few exceptions. As stated in Remark 4.1, too low and too high values of $\phi$ lead to similar performance as it can be verified from the results of $\mathrm{C} 4$ and C6. Nevertheless when $\beta_{k}$ varies fast, C6 does not give a worse performance than C4 because: i) higher values of $\phi$ allow to narrow $\mathcal{S}_{3}$ so it does not encompass $\mathcal{S}_{2}$ at the expense of increasing the probability of being outside of $\mathcal{S}_{3}$ (see Remark 6.3), ii) optimizing over $\phi$ leads to the best upperbound of the real control performance index given by $\|x\|_{\mathrm{RMS}}^{2}+\lambda\|u\|_{\mathrm{RMS}}^{2}$, but might not lead to the best solution in practice (see Remark 6.3). Finally, C8 works also better than $\mathrm{C} 4$ when $\beta_{k}$ varies fast because it follows more accurately PAR changes without enlarging much $\mathcal{S}_{3}$.

Table II. Control performance index $J_{k}^{c}$ for different PAR scenarios at time 300sec with $x_{0}=\left[\begin{array}{lll}4 & 4 & 4\end{array}\right]^{T}$.

\begin{tabular}{|c|c|c|c|c|c|c|c|c|c|}
\hline Changes $\beta_{k}$ & $\mathrm{C} 1$ & $\mathrm{C} 2$ & $\mathrm{C} 3$ & $\mathrm{C} 4$ & $\mathrm{C} 5$ & $\mathrm{C} 6$ & $\mathrm{C} 7$ & $\mathrm{C} 8$ & C9 \\
\hline$\beta_{k}=0.4$ & 0.051 & 0.041 & 0.033 & 0.029 & 0.033 & 0.031 & 0.040 & 0.030 & 0.040 \\
$100 \mathrm{sec}$ & 0.048 & 0.038 & 0.026 & 0.017 & 0.026 & 0.022 & 0.022 & 0.019 & 0.022 \\
$5 \mathrm{sec}$ & 0.053 & 0.042 & 0.032 & 0.030 & 0.032 & 0.029 & 0.034 & 0.026 & 0.033 \\
\hline
\end{tabular}

In conclusion, this example shows that:

i) Parameter $a$ should be tuned according to a priori information about the behaviour of the real PAR (see Remark 2.1 and Remark 4.2). If no information is available it is recommended to choose higher values to narrow $\mathcal{S}_{3}$.

ii) Parameter $\phi$ could also be selected considering the evolution of the PAR (see Remark 6.3), but a simple solution is to choose the one the minimizes the cost index of the optimization-based design problem.

iii) Focusing on performance optimization just on the steady state behaviour of the PAR and scheduling both the observer and controller gains with the estimated PAR helps to increase the control performance. 


\section{CONCLUSIONS}

In the present paper, we designed an observer-based controller to work under packetized control input and measurement transmissions subject to dropouts with an uncertain time-varying PAR, but with successful delivery acknowledgement.

Making use of the data reception states, we proposed a filter that gives an estimation of the real time PAR with a bounded estimation error during PAR steady states. Then, we derived a gain-scheduling strategy for the design of the state observer and controller depending of rational functions of the estimated PAR. The controller not only depends on the estimated state but also on the previous control input. Applying the separation principle, both designs are performed by means of a parametrized optimization problem that minimizes the performance index level (RMS norm of the state estimation error for the state observer, or RMS norm of the closed loop state for the controller) as a function of the possible constant PAR while guaranteeing certain robustness against PAR estimation errors and variations of the PAR. To address the numerical solution of the optimization problem we employed SOS decomposition techniques leading to an optimization problem over polynomials. Numerical examples studied the tuning of the different parameters and showed the effectiveness of the proposal.

Future work might include the extension of the current results to the case of transmissions without successful delivery acknowledgement.

\section{ACKNOWLEDGEMENT}

This work has been supported by MICINN project number TEC2015-69155-R from the Spanish government, project number P1·1B2015-42 and grant PI15734 from Universitat Jaume I.

\section{REFERENCES}

1. Hespanha JP, Naghshtabrizi P. A survey of recent results in networked control systems. Proceedings of the IEEE 2007; 95(1): 138-162.

2. Smith S, Seiler P. Estimation with lossy measurements: jump estimators for jump systems. IEEE Transactions on Automatic Control 2003; 48(12):2163-2171.

3. Morais CF, Braga MF, Oliveira RCLF, Peres PLD. $H_{\infty}$ and $H_{2}$ control design for polytopic continuous-time Markov jump linear systems with uncertain transition rates. International Journal of Robust and Nonlinear Control 2015

4. Wang J, Zhang Q, Yan XG, Zhai D. Stochastic stability and stabilization of discrete-time singular Markovian jump systems with partially unknown transition probabilities. International Journal of Robust and Nonlinear Control 2015; 25(10):1423-1437.

5. Fioravanti AR, Gonçalves APC, Geromel JC. Discrete-time $H_{\infty}$ output feedback for Markov jump systems with uncertain transition probabilities. International Journal of Robust and Nonlinear Control 2013; 23(8):894-902.

6. Zorzi M, Rao RR, Milstein LB. Error statistics in data transmission over fading channels. IEEE Transactions on Communications 1998; 46(11):1468-1477.

7. Zhu Y, Zhang L, Basin MV. Nonstationary $H_{\infty}$ dynamic output feedback control for discrete-time Markov jump linear systems with actuator and sensor saturations. International Journal of Robust and Nonlinear Control 2015; .

8. Yin Y, Shi P, Liu F, Teo KL. Observer-based $H_{\infty}$ control on nonhomogeneous Markov jump systems with nonlinear input. International Journal of Robust and Nonlinear Control 2014; 24(13):1903-1924.

9. Wang Z, Shen B, Liu X. $H_{\infty}$ filtering with randomly occurring sensor saturations and missing measurements. Automatica 2012; 48(3):556-562.

10. Liang Y, Chen T, Pan Q. Optimal linear state estimator with multiple packet dropouts. IEEE Transactions on Automatic Control 2010; 55(6):1428-1433.

11. Seiler P, Sengupta R. An $H_{\infty}$ approach to networked control. IEEE Transactions on Automatic Control 2005; 50:356-364.

12. Schenato L, Sinopoli B, Franceschetti M, Poolla K, Sastry SS. Foundations of control and estimation over lossy networks. Proceedings of the IEEE 2007; 95(1):163-187.

13. Lacerda MJ, Tognetti ES, Oliveira RCLF, Peres PLD. A new approach to handle additive and multiplicative uncertainties in the measurement for LPV filtering. International Journal of Systems Science 2014; .

14. Sato M, Peaucelle D. Gain-scheduled output-feedback controllers using inexact scheduling parameters for continuous-time LPV systems. Automatica 2013; 49(4):1019-1025.

15. Heemels WPMH, Daafouz J, Millerioux G. Observer-based control of discrete-time LPV systems with uncertain parameters. IEEE Transactions on Automatic Control 2010; 55(9):2130-2135.

16. Daafouz J, Bernussou J, Geromel JC. On inexact LPV control design of continuous-time polytopic systems. IEEE Transactions on Automatic Control 2008; 53(7):1674-1678. 
17. Luo Y, Wei G, Ding X, Liu Y. Controller design for 2-D stochastic nonlinear Roesser model: A probabilitydependent gain-scheduling approach. Journal of the Franklin Institute Nov 2014; 351(11):5182-5203.

18. Wei G, Wang Z, Shen B. Probability-dependent gain-scheduled control for discrete stochastic delayed systems with randomly occurring nonlinearities. International Journal of Robust and Nonlinear Control 2013; 23(7):815-826.

19. Wei G, Wang Z, Shen B, Li M. Probability-dependent gain-scheduled filtering for stochastic systems with missing measurements. IEEE Transactions on Circuits and Systems II: Express Briefs 2011; 58(11):753-757.

20. Peñarrocha I, Dolz D, Sanchis R. A polynomial approach for observer design in networked control systems with unknown packet dropout rate. 52nd IEEE Conference on Decision and Control, 3, 2013; 5933-5938.

21. Chesi G. LMI techniques for optimization over polynomials in control: a survey. IEEE Transactions on Automatic Control 2010; 55(11):2500-2510.

22. Moayedi M, Foo YK, Soh YC. Networked LQG control over unreliable channels. International Journal of Robust and Nonlinear Control 2013; 23(2):167-189.

23. Gawthrop P, Wang L. The system-matched hold and the intermittent control separation principle. International Journal of Control 2011; 84(12):1965-1974.

24. Wu D, Wu J, Chen S. Separation principle for networked control systems with multiple-packet transmission. IET Control Theory \& Applications 2011; 5(3):507.

25. Dolz D, Peñarrocha I, Sanchis R. Jump state estimation with multiple sensors with packet dropping and delaying channels. International Journal of Systems Science 2015; 47(4):982-993.

26. Schenato L, Sinopoli B, Franceschetti M, Poolla K, Sastry SS. Foundations of Control and Estimation Over Lossy Networks. Proceedings of the IEEE 2007; 95(1):163-187.

27. Dolz D, Peñarrocha I, Sanchis R. Networked gain-scheduled fault diagnosis under control input dropouts without data delivery acknowledgment. International Journal of Robust and Nonlinear Control 2016; 26(4):737-758.

28. Quevedo DE, Ahlén A, Leong AS, Dey S. On kalman filtering over fading wireless channels with controlled transmission powers. Automatica 2012; 48(7):1306-1316. 